\title{
Global sensitivity analysis with 2D hydraulic codes: Applied protocol and practical tool
}

\author{
M. Abily*, N. Bertrand† O. Delestre $\ddagger$ P. Gourbesville§ \\ Y. Richet" and C.-M. Duluc ${ }^{\|}$
}

October 11, 2018

\begin{abstract}
Global Sensitivity Analysis (GSA) methods are useful tools to rank input parameters uncertainties regarding their impact on result variability. In practice, such type of approach is still at an exploratory level for studies relying on 2D Shallow Water Equations (SWE) codes as GSA requires specific tools and deals with important computational capacity. The aim of this paper is to provide both a protocol and a tool to carry out a GSA for 2D hydraulic modelling applications. A coupled tool between Prométhée (a parametric computation environment) and FullSWOF_2D (a code relying on 2D SWE) has been set up: Prométhée-FullSWOF_2D (P-FS). The main steps of our protocol are: i) to identify the $2 \mathrm{D}$ hydraulic code input parameters of interest and to assign them a probability density function, $i$ i) to propagate uncertainties within the model, and iii) to rank the effects of each input parameter on the output of interest. For our study case, simulations of a river flood event were run with uncertainties introduced through three parameters using P-FS tool. Tests were performed on regular computational mesh, spatially discretizing an urban area, using up to 17.9 million of computational points. P-FS tool has been installed on a cluster for computation. Method and P-FS tool successfully allow the computation of Sobol indices maps.

Keywords Uncertainty, flood hazard modelling, global sensitivity analysis, 2D shallow water equation, Sobol index.

Analyse globale de sensibilité en modélisation hydraulique à surface libre 2D : application d'un protocole et développement d'outils opérationnels Les méthodes d'analyse de sensibilité permettent de contrôler la robustesse des résultats de modélisation ainsi que d'identifier le degré d'influence des paramètres d'entrée sur le résultat en sortie d'un modèle. Le processus complet constitue une analyse globale de sensibilité (GSA). Ce type d'approche présente un grand intérêt pour analyser les incertitudes de résultats de modélisation, mais est toujours à un stade exploratoire dans les études appliquées mettant en jeu des codes basés sur la résolution bidimensionnelle des équations de Saint-Venant. En effet, l'implémentation d'une GSA est délicate car elle
\end{abstract}

*Polytech'Nice Sophia \& URE Innovative-CiTy, University of Nice Sophia Antipolis, France, e-mail : abily@polytech.unice.fr

${ }^{\dagger}$ Institut de Radioprotection et de Sûreté Nucléaire (IRSN), PRP-DGE, SCAN, BEHRIG, France, e-mail : nathalie.bertrand@irsn.fr

${ }^{\ddagger}$ Lab. J.A. Dieudonné UMR7351 CNRS \& EPU Nice Sophia, University of Nice, France, e-mail : delestre@math.unice.fr

§Polytech'Nice Sophia \& URE Innovative-CiTy, University of Nice Sophia Antipolis, France

『Institut de Radioprotection et de Sret Nuclaire (IRSN), PSN-EXP, SNC, France

"Institut de Radioprotection et de Sûreté Nucléaire (IRSN), PRP-DGE, SCAN, BEHRIG 
nécessite des outils de paramétrage automatisable spécifique et requiers d'importante capacité de calcul. L'objectif de cet article est de présenter un protocole et des outils permettant la mise en œuvre d'une GSA dans des applications de modélisation hydraulique 2D. Un environnement paramétrique de calcul (Prométhée) et un code de calcul basé sur les équations de Saint-Venant 2D (FullSWOF_2D) ont été adaptés pour développer l'outil Prométhée-FullSWOF_2D (P-FS). Un prototype de protocole opérationnel pour la conduite d'une GSA avec un code de calcul d'hydraulique à surface libre $2 \mathrm{D}$ est présenté et appliqué à un cas test de crue fluvial en milieu urbain. Les étapes du protocole sont: $i$ ) l'identification des paramètres hydrauliques $2 \mathrm{D}$ d'intérêt et l'attribution d'une loi de probabilité aux incertitudes associées à ces paramètres, ii) la propagation des incertitudes dans le modèle, et iii) le classement des effets des incertitudes des paramètres d'entrées sur la variance de la sortie d'intérêt. Pour le cas testé, des simulations d'un scénario ont été effectuées avec une incertitude portée sur trois paramètres d'entrée. L'outil P-FS a été utilisé sur un cluster de calcul et est aisément transposable sur d'autres architectures de calcul intensif. Le protocole de GSA et P-FS ont permis de produire des cartes d'indices de Sobol afin d'analyser la variabilité spatiale des contributions des paramètres incertains.

Mots clés Incertitude, modélisation d'inondation extrême, méthode d'analyse de sensibilité, équations de Saint-Venant 2D, indices de Sobol.

\section{Introduction}

Sensitivity analysis is an important aspect of the responsible use of hydraulic models. Such approach allows the identification of the key parameters impacting model performance [Tekatlian, 2001; Iooss, 2011]. Global Sensitivity Analysis (GSA) aims at ranking the input parameters variability effects on models output variability. Only few studies have performed GSA on hydraulic models. Indeed, this type of approach is not straight forward, as it requires adaptation of methods and tools development. These aspects being time consuming, not without standing the heavy computational cost of such type of approach, it represents an important investment for applied practitioners community and is therefore still at an exploratory level.

Nevertheless, dealing with uncertainties in hydraulic models is a press-forward concern for both practitioners [Iooss, 2011] and new guidance [ASN, 2013]. In practical flood event modelling applications, mostly uses standard industrial codes relying on Shallow Water Equations (SWEs) either in 1D (e.g. Mascaret [Goutal et al., 2012], Mike 11 [DHI, 2009] etc.) or 2D (e.g. Telemac 2D [Hervouet, 1999], Mike 21 [DHI, 2007], Isis2D [Halcrow, 2012] etc.). The aim of using hydraulic models is to provide information on simulated flood event properties such as maximal water depth or flood spatial extent. Eventually outputs of the models are used for design or safety assessment purpose.

In hydraulics, deterministic mathematical models aim at representing natural phenomena with different levels of complexity in the mathematical formulation of the physical phenomena depending on underlying simplifying assumptions. These assumptions will influence the domain of validity and of application of the models. Consequently, it will impact accuracy standards which should be expected from models results. An analytical solution to SWE exists from a mathematical point of view only when the problem is well-posed, which is generally not the case in practical river flood modelling engineering applications [Cunge, 2014]. Moreover, equations are resolved using computer codes, which will discretely approach the continuous solutions of these equations (when mathematically existing). Numerical approach implemented in these codes can be various and different level of accuracy can be expected depending on the numerical method. In deterministic hydraulic codes, input parameters are 
variables which are known with a certain level of confidence. Eventually, modeller choices to design models and computation optimization can introduce high variability in results. In hydraulic models, sources of uncertainties can be classified in three categories: $(i)$ hypothesis in mathematical description of the natural phenomena, (ii) numerical aspects when solving the equations, and (iii) input parameters of the model. The uncertainties related to input parameters are of prime interest for applied practitioners willing to decrease uncertainties in theirs models results.

Hydraulic models input parameters are either of hydrological, hydraulic, topographical or numerical type. Identification, classification and impact quantification of sources of uncertainties, on a given model output, are a set of analysis steps which will enable to $(i)$ analyze uncertainties behavior in a given modelling problem, $(i i)$ elaborate methods for reducing uncertainties on a model output, and ( iii) communicate on relevant uncertainties. Uncertainty Analysis (UA) and Sensitivity Analysis (SA) methods are useful tools, as they allow robustness of model predictions to be checked and help to identify input parameters influences. UA consists in the propagation of uncertainty sources through the model, and then focus on the quantification of uncertainties in model output and propagating them through the model predictions [Saint-Geours, 2012]. It allows robustness of model results to be checked. Various methods are then available to rank parameters regarding their impact on results variability (such as Sobol index [Sobol', 1990]). This process goes one step beyond UA and constitutes a global sensitivity analysis (GSA). In practice, such type of approach is of a great interest, but is still at an exploratory level in applied studies relying on 2D SWE codes. Indeed, GSA approach implementation is challenging, as it requires specific tools and deals with important computational capacity. With 1D free surface hydraulic codes, applied tools and methodology for uncertainty propagation and for GSA have been developed by IRSN and Companie Nationale du Rhône (CNR) [Nguyen et al., 2015].

The purpose of the study presented in this paper is to apply a protocol and to provide a tool, allowing adaptable and ready-to use GSA for 2D hydraulic modelling applications. Sections developed in this paper present the concept of GSA applied to 2D hydraulic modelling approach through the presentation $(i)$ of the GSA concept, (ii) of implemented protocol and (iii) of developed operational tools. A proof of concept to illustrate feasibility of the approach is given, based on a 2D flood river event modelling in Nice (France) low Var valley.

\section{Material and methods}

\subsection{Global Sensitivity Analysis approach}

A regular sensitivity analysis aims to study how the variations of model input parameters impact the models outputs. Objectives with this approach are mostly to identify the parameter or set of parameters which significantly impact models outputs [Volkova et al., 2008; Marrel et al., 2012]. For instance, SA screening approach is a variance-base method which allows to identify input variables which have negligible effects, from those which have linear, non-linear or combinatory effects significantly impacting variability of results output. This can be useful for models with a large set of input parameters assumed to introduce uncertainty, to reduce the number of input parameters to consider in a given SA study. Local SA focuses on fixed point in the space of the input. The aim is here to address model behavior near parameters nominal value to safely assume local linear dependence on the parameter. More details about these SA methods and their application to practical engineering problems can be found in [Iooss, 2011; Saltelli et al., 2000; Jacques, 2011].

A GSA aims to quantify the output uncertainty in the input factors, given by their uncer- 
tainty range and distribution [Volkova et al., 2008]. To do so, the deterministic code (2D hydraulic code in our case) is considered as a black box model as described in [Marrel et al., 2012]:

$$
\begin{aligned}
f: & \mathbb{R}^{p} \rightarrow \mathbb{R} \\
& X \mapsto Y=f(X)
\end{aligned}
$$

where $f$ is the model function, $X=\left(X_{1} ; \ldots ; X_{p}\right)$ are $p$ independent input random variables with known distribution and $Y$ is the output random variable. The principle of GSA method relies on estimation of inputs variables variance contribution to output variance. A unique functional analysis of variance (ANOVA) decomposition of any integral function into a sum of elementary functions allows to define the sensitivity indices as explained in [Sobol', 1990; Marrel et al., 2012; Saint-Geours, 2012]. Sobols indices are defined as follow:

$$
S_{i}=\operatorname{Var}\left[\mathbb{E}\left(Y \mid X_{i}\right)\right] / \operatorname{Var}(Y) .
$$

To implement a GSA approach, it is necessary $(i)$ to identify inputs and assess their probability distribution, (ii) to propagate uncertainty within the model and (iii) to rank the effects of input variability on the output variability through functional variance decomposition method such as calculation of Sobol indices. The first two steps constitute an uncertainty analysis (UA) which is a compulsory stage of the GSA.

For the first step of the UA, each input parameter is considered as a random value where both, choice of considered input parameter and choice of probability distribution of the input random values, have to be set up. The assessment of selected uncertain parameters and their probability distribution is completed according to expert opinion or by statistical analysis for measured values if sufficient measured data sets are available. The first two steps lead to define probability density function constructed to represent uncertainty of selected input parameters for the study. It has to be emphasized that this step of the GSA process is important and strongly subjective [Volkova et al., 2008].

Propagation of uncertainty is then required (step $i i$ of the UA), all sources of uncertainties are varied simultaneously, which is classically done using Monte-Carlo techniques or more parsimonious Monte-Carlo like approach [Iooss, 2011]. Controlling the convergence of the uncertainty propagation gives an idea if the sample of simulations is large enough to allow consistent statistical analysis. In practice, convergence of estimated sensitivity indices and their confidence interval can be plotted and examined visually [Marrel et al., 2012]. Nevertheless, the decision whether the level of convergence is satisfactory or not, depends on arbitrary decision of the operator regarding desired accuracy and confidence interval on the accuracy. Eventually, GSA can be performed to calculate Sobol index (2). First-order Sobol index indicates the contribution to the output variance of the main effect of each input parameters. Total-order Sobol index measures the contribution to the output variance including all variance caused by interactions between uncertain input parameters [Iooss, 2011]. The production of Sobol index spatial distribution map is promising. Moreover, such maps have been done in other application fields such as hydrology, hydrogeology and flood risk cost estimation [Saint-Geours, 2012].

\subsection{Protocol applied for GSA with 2D hydraulic models}

An overview of GSA approach is presented in fig. 1. This type of general protocol for GSA has already been applied to 1D hydraulic model [Iooss, 2011; Nguyen et al., 2015; Alliau et al., 2015]. Common aspects arise between 1D and 2D models hydraulic application to implement a GSA approach and are described step by step below:

The first step (Step $i$, in fig. 1), identifies the input parameters of the hydraulic code to 


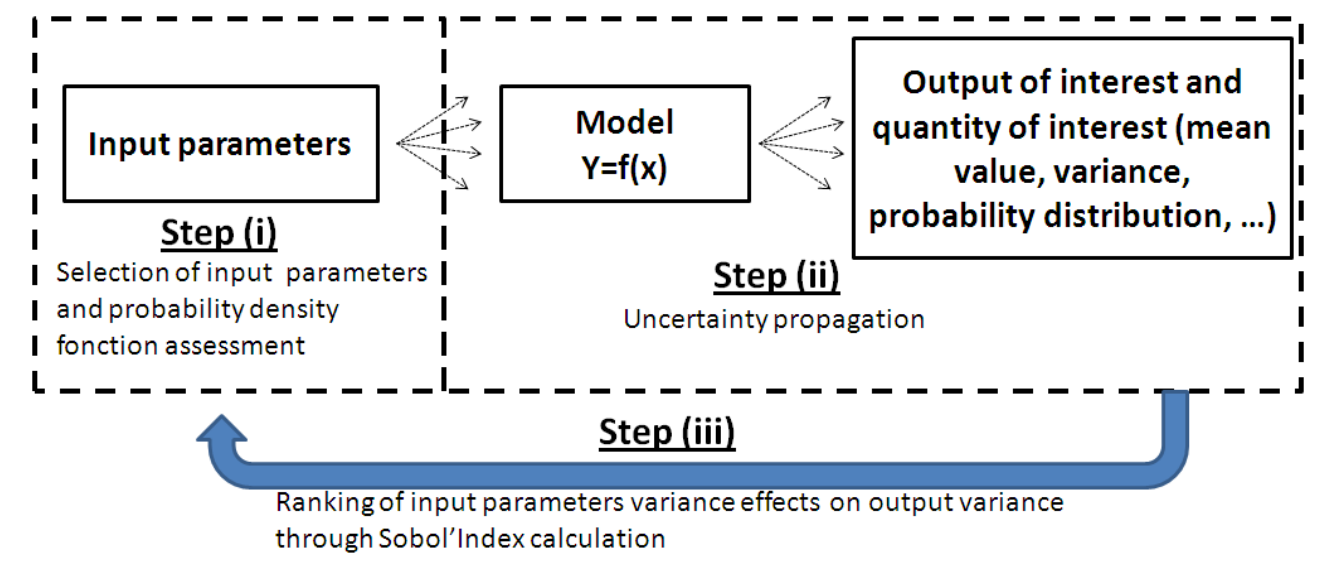

Figure 1: Standard GSA approach.

be considered for the analysis. In hydraulic models, the mains input parameters to consider are: $i)$ geometric parameters, spatial discretization, hydraulic and numerical parameters.

The geometric parameters, in 1D models, are represented as cross section, whereas in 2D models Digital Elevation Model (DEM) are included as component of geometric parameter. Another important geometrical aspect to consider as an input parameter introducing uncertainty is the spatial discretization. The spatial discretization can be considered as a geometrical and a numerical parameter. Indeed spatial discretization impacts both the level of details of the geometry included in calculation and numerical stability of calculation as, $d x$ (and $d y$ in 2D) impact CFL criterion. Hydraulic parameters are mainly initial, boundary conditions and energy loses coefficient. Such parameters are introduced in models under the form of water level, discharge or flow velocity field or friction coefficients. Geometric and hydraulic variables can be measured or estimated; as a result they are often subject to a high level of uncertainty.

Numerical parameters are related to numerical method and solver included in numerical scheme. Broadly speaking, input numerical parameters will be those related to CFL number $(d t$ and $d x$ ), parameters impacting accuracy of solver (numbers of iteration or order of solver method for instance) as well as parameters impacting numerical diffusion and dispersion.

Once input parameters are selected, a probability density function has to be attributed to each parameter, in order to distribute input parameters. As previously mentioned, probability density function are most often related to expert opinion. The most common used distributions in hydraulic studies are normal or triangular distribution [Nguyen et al., 2015]. Uniform distribution is used as well, when no clear idea can be made up regarding the distribution of a given parameter variability. By assuming equi-probability of the realization $p$ of a variable, uniform distribution will then maximize the uncertainty (entropy) of the input parameter.

To apply a GSA, the input parameters are assumed to be independent. This specific point should be considered at this stage of the GSA, as sometimes selected input parameters can be strongly correlated in some cases. For example, in hydraulic model dependences between flow and linear energy loses properties (represented by Chézy, Manning or Strickler law) are present.

The second step (Step ii, in fig. 1) results in the propagation of the distributed input parameters within the selected hydraulic model. Hydraulic codes (1D and 2D) are based on 
the same sets of equations which are the SWE (3), as written as follow in 2D [Cunge et al., 1980]:

$$
\left\{\begin{array}{l}
\partial_{t} h+\partial_{x}(h u)+\partial_{y}(h v)=0, \\
\partial_{t}(h u)+\partial_{y}\left(h u^{2}+g h^{2} / 2\right)+\partial_{y}(h u v)=g h\left(S_{0 x}-S_{f_{x}}\right), \\
\partial_{t}(h v)+\partial_{y}(h u v)+\partial_{y}\left(h v^{2}+g h^{2} / 2\right)=g h\left(S_{0 y}-S_{f_{y}}\right),
\end{array}\right.
$$

where the unknowns are the velocities $u(x, y, t))$ and $v(x, y, t)[\mathrm{m} / \mathrm{s}]$ and the water height $h(x, y, t)[m]$, and where $S_{0 x}\left(S_{0 y}\right)$ is the opposite of the slope and $S_{f_{x}}\left(S_{f_{y}}\right)$ the linear energy losses in $x$-direction (resp. $y$-direction).

Therefore, underlying hypothesis in the mathematical description of the physical process are similar for 1D and 2D models: (i) uniform $u$ (and uniform $v$ in 2D) velocity for a given mesh cell, (ii) horizontal free surface at a given cell, (iii) vertical hydrostatic pressure and (iv) energy losses are represented through Chézy, Manning or Strickler formulas [Cunge et al., 1980; Chow, 1959]. As previously mentioned, the model is considered as a black box as described concerning (1) for application of the GSA.

For each specific source of uncertainty, $n$ independent realizations are generated using MonteCarlo techniques. The number of realizations has to be large enough to reach convergence of the interest variable. Histograms are commonly plotted to ensure that output parameters follow a normal distribution. Moreover, spatially distributed results of the variable of interest can then be analyzed.

The third step (Step iii, in fig. 1) and final step of GSA approach is the calculation of the Sobol indices and the evaluation of the model outputs robustness. Analysing GSA results, the model user has a better understanding and quantification of its models uncertainties.

\subsection{Operational tool and setup}

To apply a GSA with 2D Hydraulic models, a coupling between Prométhée a code allowing a parametric environment of other codes, has been performed with FullSWOF_2D, a twodimensional SWE based hydraulic code. The coupling procedure has taken advantage of previous coupling experience of Promthe with 1D SWE based hydraulic code [Nguyen et al., 2015; Alliau et al., 2015]. The coupled code Prométhée-FullSWOF (P-FS) has been performed on a HPC computation structure.

\subsubsection{FullSWOF_2D}

FullSWOF_2D (Full Shallow Water equation for Overland Flow in 2 dimensions) is a code developed as a free software based on 2D SWE [Delestre et al., 2014]. In FullSWOF_2D, the 2D SWE are solved thanks to a well-balanced finite volume scheme based on the hydrostatic reconstruction [Audusse et al., 2004; Delestre, 2010]. The finite volume scheme, which is suited for a system of conservation low, is applied on a structured spatial discretization, using regular Cartesian mesh. For the temporal discretization, a variable time step is used based on the CFL criterion. The hydrostatic reconstruction (which is a well-balanced numerical strategy) allows to ensure that the numerical treatment of the system preserves water depth positivity and does not create numerical oscillation in case of a steady states, where pressures in the flux are balanced with the source term here (topography). Different solvers can be used HLL, Rusanov, Kinetic [Bouchut, 2004], VFROE combined with first order or second order (MUSCL or ENO) reconstruction.

FullSWOF_2D is an object oriented software developed in $\mathrm{C}++$. Two parallel versions of the code have been developed allowing to run calculations under HPC structures [Cordier, S. et al., 2013]. 


\subsubsection{Prométhée-FullSWOF}

Prométhée software is coupled with FullSWOF_2D. Prométhée is an environment for parametric computation, allowing carrying out uncertainties propagation study, when coupled to a code. This software is an open source environment developed by IRSN (http:// promethee.irsn.org/doku.php). The main interest of Prométhée is the fact that it allows the parameterization of any numerical code. Also, it is optimized for intensive computing resources use. Moreover, statistical post-treatment can be performed using Prométhée as it integrates R statistical computing environment [Ihaka, 1998]. The coupled code Prométhée/FullSWOF (P-FS) is used to automatically launch parameterized computation through R interface under Linux OS. A graphic user interface is available under Windows OS, but in case of large number of simulation launching, the use of this OS has shown limitations as described in [Nguyen et al., 2015]. A maximum of 30 calculations can be run simultaneously, with the use of 30 daemons.

\subsection{Practical application test case}

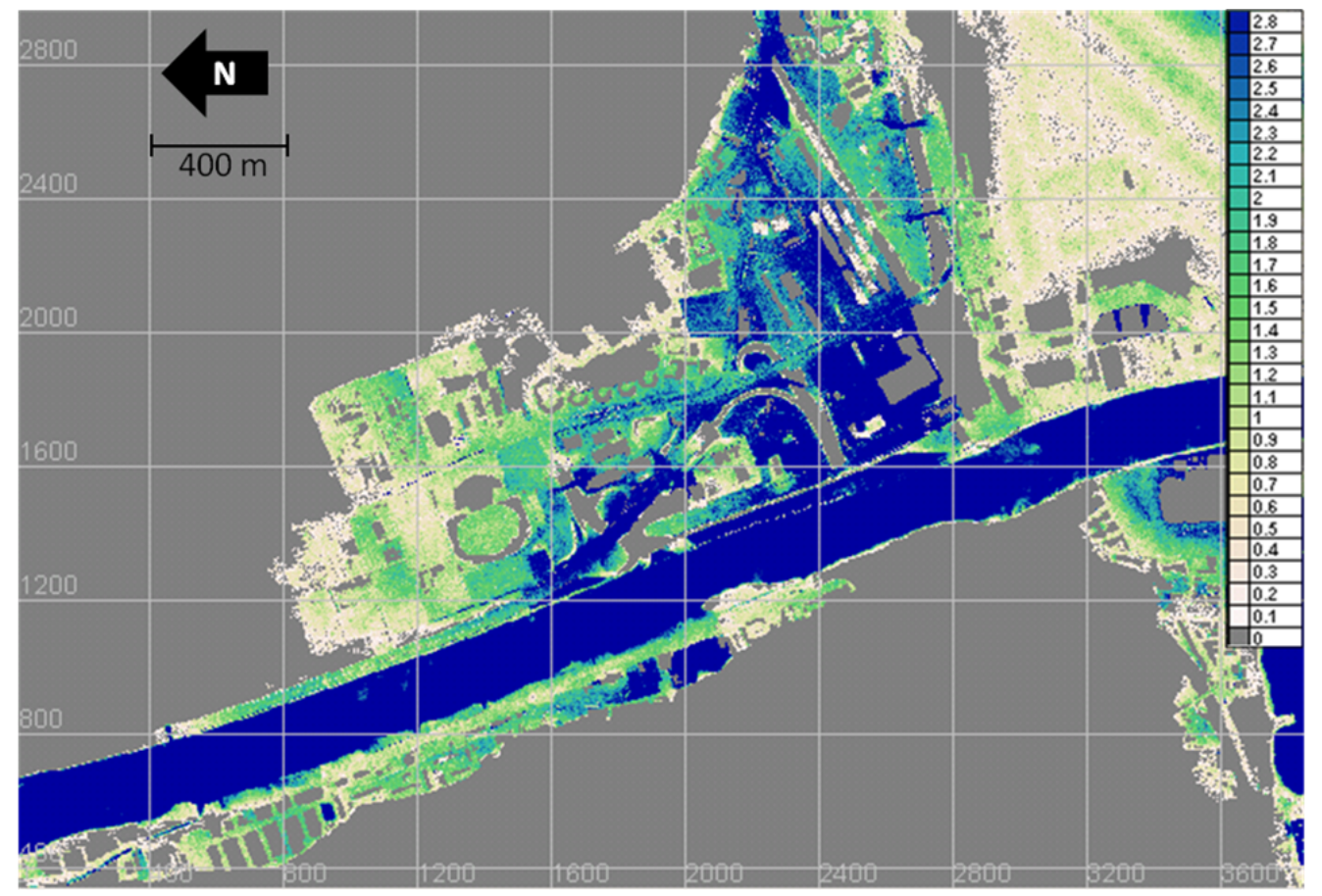

Figure 2: Illustration of $h_{\max }$ map during an extreme flood event scenario simulated for the low Var river valley using FullSWOF_2D.

To test the GSA protocol and P-FS tools, uncertainties introduced in 2D hydraulic models by geometric input parameters related to HR topographic data use have been studied. More specifically this case focuses on uncertainty related to 3D classified data use within hydraulic 2D models. In [Abily et al., 2016], the case study is introduced and the outcomes of the GSA applied to uncertainties related to high resolution topographic data use with $2 \mathrm{D}$ hydraulic codes are presented in detail. It has to be reminded that the scope of present paper is to give 
a proof of concept of possibility to apply protocol and to use developed tools for a GSA with 2D SWE based hydraulic codes. The main characteristics of that case study are summarized in the current section.

The 1994 flood event of the Var river valley has been modelled. The study area is the lower part of the Var river valley, where major inundation occurred during the 1994 event. As our analysis focus on uncertainties related to geometric input parameters, hydrologic and hydraulic parameters are treated as constant. An illustration of the maximal water depth $\left(h_{\max }\right)$ computed in this area for the given hydraulic scenario is illustrated in fig. 2 .
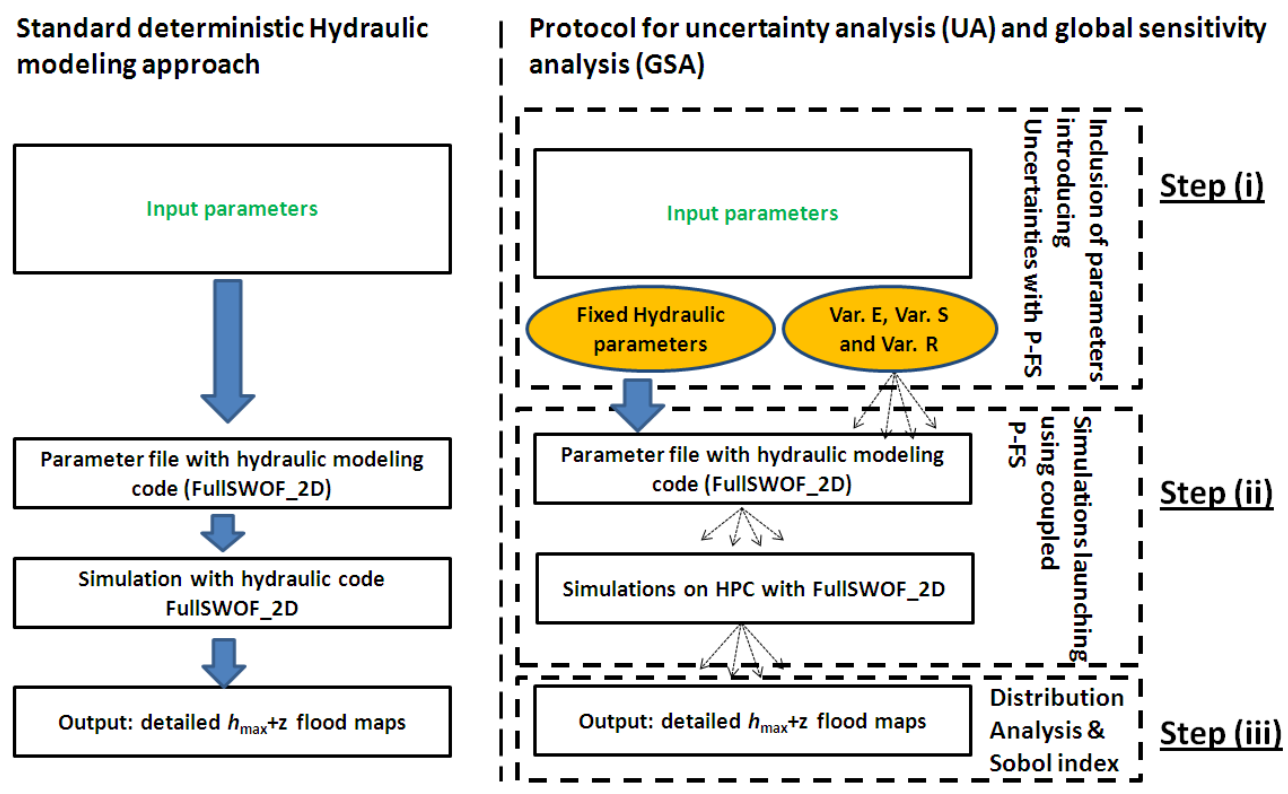

Figure 3: Schematization of the GSA protocol adapted to 2D hydraulic modelling.

Fig. 3 illustrates the GSA approach adapted to the tested proof-of-concept 2D hydraulic modelling study. The three input parameters related to the geometric input parameters are $(i)$ the topographic input (called Var. $E$ ) and (ii) two numerical parameters chosen by modellers (called Var. $S$ and Var. $R$ ) when willing to use HR 3D classified data are considered in this GSA practical case.

Var. $E$ represents measurement errors in topography. Var. $S$ and Var. $R$, are discrete values representing operator choices, which are respectively concrete elements structures included in DEM (buildings, walls, street elements etc.) and structured mesh resolution. These three parameters Var. E, $S$ and $R$, are assumed independent. Var. $E$ is 100 different occurrences of spatially homogenous $1 \mathrm{~m}$ resolution maps of errors where each cell of the error grids follows a normal probability density function $\mathcal{N}(0 ; 0.2)$. A given occurrence of Var. $E$ is the addition of one of the error grid to a reference High resolution DTM. Var. $S$ can have 4 discrete values depending on modeler choices when including HR concrete above ground elements to DEM. The $S 1$ stands for a row DTM, $S 2$ for a DEM which encompasses building elevation information, $S 3$ stands for a DEM including $S 2$ elevation information plus concrete walls elevations and eventually, $S 4$ is a DEM which including $S 3$ information plus Concrete street elements elevation information. Var. $R$ can have 5 discrete values being $1,2,3,4$ or 
$5 \mathrm{~m}$, representing spatial discretization choice.

As the number of considered parameters is limited, SA screening methods are not considered. The output of interest is the overland flow water surface elevation $\left(h_{\max }+z\right)$ reached at points and areas of interest. The GSA ranks influence of selected input parameters variability over variability of $h_{\max }+z$.

\section{Results and discussion}

\subsection{Feasibility and operational achievement of the approach}

The introduced GSA approach has been followed with a two-dimensional hydraulic model. The coupled tool has been set up on the HPC Mésocentre and the P-FS couple is transposable on any HPC structures. Using R commands, the simulations were launched. However, the calculation running time of our simulation is very long and increases considerably when the mesh resolution increases. The computing CPU cost is respectively 2, 6, 12, 24, 80 hours for $5,4,3,2,1 \mathrm{~m}$ resolution grid. P-FS tool successfully allow applying GSA protocol to 2D hydraulic modelling. So far, highest resolution ( $R 1$ and $R 2)$ simulations are not fully completed yet, due to a prohibitive computational time. At these resolutions running simulations on more than $12 \mathrm{CPU}$ is necessary. Improvement of parallel version of P-FS is in progress to allow a single simulation to run over more than one node. The $\mathrm{R}$ environment allowed performing post-treatment over the results to analyze them efficiently (see section $3.2)$.

The limit of the approach is the computational time required to run the large number of simulations. In our case, simulations have been run to generate a large set of results, which were sampled afterward, using Monte-Carlo approach to calculate Sobol indices. Moreover, generation of DEM can be time consuming and cannot be entirely generated automatically following chosen probability distribution functions and especially when parameters are discrete. As a result, they have been created before the propagation has been carried out. This remark has to be extended as a current limit of the tool to generate variable spatial input for 2D hydraulic GSA. This requires further development.

\subsection{Results on the illustrative case}

The fig. 4 introduces the typical results that are obtained when a GSA is performed. Convergence test can be illustrated by the evolution of mean $h_{\max }+z$ value and the $95 \%$ confidence interval (CI) when $N$ size increases (fig. 4.a). Fig. 4.b and 4.b respectively illustrate probability density function analysis of output $\left(h_{\max }+z\right)$ when only one parameter (Var. E) is varying (fig. 4.b), and scatter plotting use for distribution analysis when two parameters (Var. $S$ and $E$ ) are varying (fig. 4.b). First-order Sobol indices are represented in fig. 4.c. Total order Sobol index can be computed as well. These results have been obtained using $\mathrm{R}$ scripts, which were written on purpose for such type of analysis using existing $\mathrm{R}$ functions. Nevertheless, it has to be emphasized that limits of the approach are $(i)$ needs to fully integrate specialization possibility in P-FS tool for spatially varying input parameters, (ii) computational time required for such an approach application over resources demanding 2D models can be prohibitive for applied practitioner and (iii) spatialization of output statistical analysis still requires few post treatment development to allow a fully holistic spatial GSA application for 2D hydraulic models. Eventually an extra round of analysis and research has to be effectuated using feedback of first results of this approach to allow improvement of different steps of GSA for 2D hydraulic models (regarding identification of parameters 


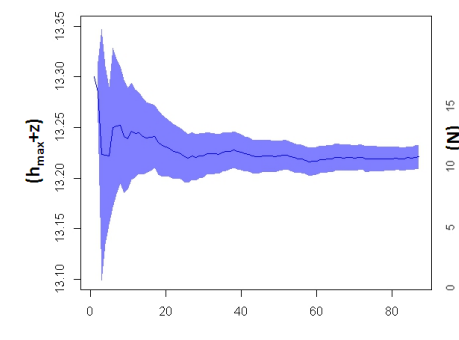

a) Convergence test

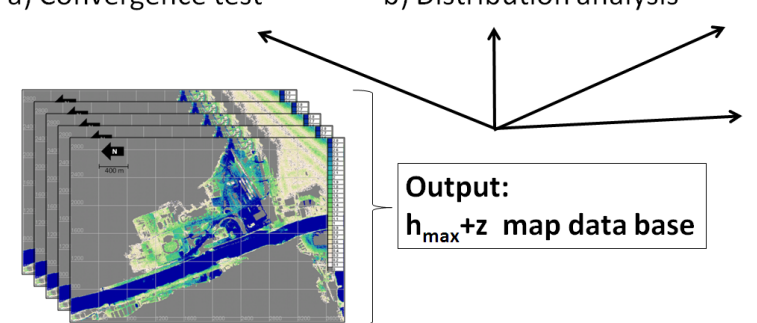

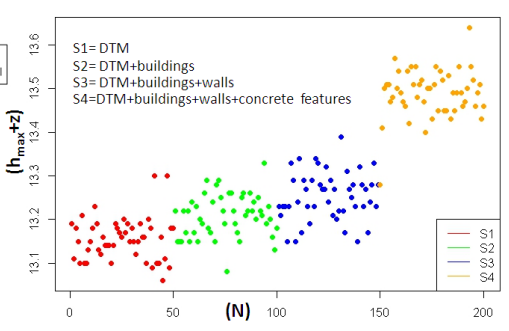

$\left.b^{\prime}\right)$ Distribution analysis

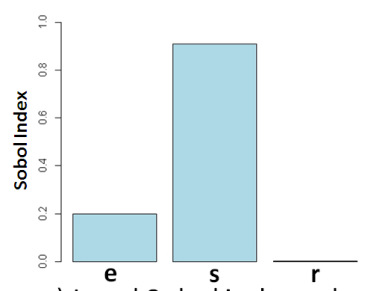

c) Local Sobol index calculation

Figure 4: Illustration of UA and GSA outputs from the proof-concept study case using protocol and developed P-FS tool.

independence for instance).

\section{Conclusions}

In this paper, a GSA framework to investigate the impact of uncertainties of deterministic 2D hydraulic models input parameters has been developed and tested over the low Var river basin. A coupled tool Prométhée-FullSWOF_2D (P-FS) has been elaborated over a standard high performance computation (HPC) structure. This tool allows parameterization and automatic launching of simulation for uncertainty propagation step of a GSA. Achieved test on low Var valley constitutes a proof of concept study which has put to the light the promising possibilities of such an approach for identification of most influent uncertain input parameters. Indeed, it is possible to go all the way through GSA protocol and after convergence checking, ranking of influent uncertain input parameter is possible. P-FS is ready to use and easily compatible with most of HPC structures. Limits and possible improvements of our protocol and tool can be emphasized as follow:

1. Generally speaking, efforts are required for characterization of input parameters spatial variability. This step of the process can be time consuming and his application in P-FS tool might not be straight forward.

2. Required computational resources to proceed to this type of study are considerable. Here, for the finest resolutions ( $R 1$ and $R 2$ ), we had to consider to increase the number of CPUs possible to use for a given simulation. This will enable to reduce running time of the simulations.

3. The next step is to carry out Sobol index map to illustrate possibilities of protocol and tool use combination with cross input parameter output variations spatial analysis. 


\section{Acknowledgments}

Photogrametric and photo-interpreted dataset used for this study have been kindly provided by Nice Côte d'Azur Metropolis for research purpose. This work was granted access to (i) the HPC and visualization resources of "Centre de Calcul Interactif" hosted by "Université Nice Sophia Antipolis" and (ii) the HPC resources of Aix-Marseille Université financed by the project Equip@Meso (ANR-10-EQPX-29-01) of the program "Investissements d'Avenir" supervised by the Agence Nationale pour la Recherche. Technical support for codes adaptation on high performance computation centers has been provided by F. Lebas., H. Coullon and P. Navarro.

\section{References}

[Abily et al., 2016] Abily, M., Delestre, O., Gourbesville, P., Bertrand, N., Duluc, C.-M., and Richet, Y. (2016). Global sensitivity analysis with 2D hydraulic codes: application on uncertainties related to high-resolution topographic data. In Gourbesville, P., Cunge, J., and Caignaert, G., editors, Advances in Hydroinformatics - Simhydro 2014, Springer Water, pages 301-315. Springer Singapore.

[Alliau et al., 2015] Alliau, D., De Saint Seine, J., Lang, M., Sauquet, E., and Renard, B. (2015). Etude du risque d'inondation d'un site industriel par des crues extrêmes: de l'évaluation des valeurs extrêmes aux incertitudes hydrologiques et hydrauliques. $L a$ Houille Blanche, 2:67-74.

[ASN, 2013] ASN (2013). Protection of Basic Nuclear Installations Against External Flooding - guide no.13 - version of 08/01/2013. Technical report, Autorité de Sûreté Nucléaire.

[Audusse et al., 2004] Audusse, E., Bouchut, F., Bristeau, M.-O., Klein, R., and Perthame, B. (2004). A fast and stable well-balanced scheme with hydrostatic reconstruction for shallow water flows. SIAM J. Sci. Comput., 25(6):2050-2065.

[Bouchut, 2004] Bouchut, F. (2004). Nonlinear stability of finite volume methods for hyperbolic conservation laws, and well-balanced schemes for sources, volume 2/2004. Birkhäuser Basel.

[Chow, 1959] Chow, V. T. (1959). Open-Channel Hydraulics. McGraw-Hill.

[Cordier, S. et al., 2013] Cordier, S., Coullon, H., Delestre, O., Laguerre, C., Le, M. H., Pierre, D., and Sadaka, G. (2013). FullSWOF Paral: Comparison of two parallelization strategies (MPI and SkelGIS) on a software designed for hydrology applications. ESAIM: Proc., 43:59-79.

[Cunge et al., 1980] Cunge, J., Holly, F., and Verwey, A. (1980). Practical Aspects of Computational River Hydraulics. Pitman Publishing, London, T. Fisher Unwin.

[Cunge, 2014] Cunge, J. A. (2014). What do we model? What results do we get? An anatomy of modelling systems foundations. In Gourbesville, P., Cunge, J., and Caignaert, G., editors, Advances in Hydroinformatics, Springer Hydrogeology, pages 5-18. Springer Singapore.

[Delestre, 2010] Delestre, O. (2010). Simulation du ruissellement d'eau de pluie sur des surfaces agricoles / rain water overland flow on agricultural fields simulation. $\mathrm{PhD}$ thesis, 
Université d'Orléans (in French), available from TEL: tel.archives-ouvertes.fr/INSMI/tel$00531377 /$ fr.

[Delestre et al., 2014] Delestre, O., Cordier, S., Darboux, F., Du, M., James, F., Laguerre, C., Lucas, C., and Planchon, O. (2014). FullSWOF: A software for overland flow simulation. In Gourbesville, P., Cunge, J., and Caignaert, G., editors, Advances in Hydroinformatics, Springer Hydrogeology, pages 221-231. Springer Singapore.

[DHI, 2007] DHI (2007). MIKE 21 FLOW MODEL, Hydrodynamic Module - Scientific Documentation. Technical report, Danish Hydraulics Institute, Horsholm, Denmark.

[DHI, 2009] DHI (2009). MIKE 11, A Modelling System for Rivers and Channel - Reference Manual. Technical report, Danish Hydraulics Institute, Horsholm, Denmark.

[Goutal et al., 2012] Goutal, N., Lacombe, J.-M., Zaoui, F., and El-Kadi-Abderrezak, K. (2012). MASCARET: a 1-D open-source software for flow hydrodynamic and water quality in open channel networks. In Murillo Muñoz, R., editor, River Flow 2012: Proceedings of the International Conference on Fluvial Hydraulics, San José, Costa Rica, 5-7 September - Volume 2, pages 1169-1174. CRC Press.

[Halcrow, 2012] Halcrow (2012). ISIS 2D quick start guide - v. 3.6. Technical report.

[Hervouet, 1999] Hervouet, J.-M. (1999). Telemac, a hydroinformatic system/ Télémac, un système hydroinformatique. La Houille Blanche, 3-4:21-28.

[Ihaka, 1998] Ihaka, R. (1998). R: Past and future history. A Draft of a Paper for Interface '98.

[Iooss, 2011] Iooss, B. (2011). Revue sur l'analyse de sensibilité globale de modèles numériques. Journal de la Société Française de Statistique, 152(1):1-23.

[Jacques, 2011] Jacques, J. (2011). Pratique de l'analyse de sensibilité : comment évaluer l'impact des entrées aléatoires sur la sortie d'un modèle mathématique. http://math.univlille1.fr/ jacques.

[Marrel et al., 2012] Marrel, A., Iooss, B., Veiga, S., and Ribatet, M. (2012). Global sensitivity analysis of stochastic computer models with joint metamodels. Statistics and Computing, 22(3):833-847.

[Nguyen et al., 2015] Nguyen, T.-m., Richet, Y., Balayn, P., and Bardet, L. (2015). Propagation des incertitudes dans les modèles hydrauliques 1D. La Houille Blanche, 5:55-62.

[Saint-Geours, 2012] Saint-Geours, N. (2012). Analyse de sensibilité de modèles spatialisés - Application à l'analyse coût-bénéfice de projets de prévention des inondations. Thèse, Université Montpellier II - Sciences et Techniques du Languedoc.

[Saltelli et al., 2000] Saltelli, A., Tarantola, S., and Campolongo, F. (2000). Sensitivity analysis as an ingredient of modeling. Statistical Science, 15(4):377-395.

[Sobol', 1990] Sobol', I. M. (1990). On sensitivity estimation for nonlinear mathematical models. Matematicheskoe modelirvanie, 2(1):112-118 (in Russian), MMCE, 1(4) (1993) :407-414 (in English).

[Tekatlian, 2001] Tekatlian, A. (2001). Etude de sensibilité des modélisations 1D: recensement et quantification des sources d'incertitude externes. Technical report, CETMEF/ CETE Méditerranée. 
[Volkova et al., 2008] Volkova, E., Iooss, B., and Van Dorpe, F. (2008). Global sensitivity analysis for a numerical model of radionuclide migration from RRC "Kurchatov Institute" radwaste disposal site. Environmental Research and Risk Assessment, 22:17-31. 